\title{
On listening where we look: The fragility of a phenomenon
}

\author{
NIGEL C. W. WOLTERS and DIANE J. SCHIANO \\ Oberlin College, Oberlin, Ohio
}

\begin{abstract}
The role of eye position information has been the subject of some debate in the literature on the visual facilitation of auditory localization and attention. In one particularly compelling study, Reisberg, Scheiber, and Potemken (1981) found that fixation position strongly influenced subjects' recall performance in a binaural selective-listening task. The present paper describes repeated failures to demonstrate the eye position effect under conditions similar to those of the original study, thus challenging the robustness of this oft-cited phenomenon of "listening where we look."
\end{abstract}

Over the past several years, a number of studies have appeared which demonstrate that viewing conditions can affect auditory localization and attention. For example, performance in sound localization tasks is better when subjects' eyes are kept open than when they are closed (Jones \& Kabanoff, 1975; Warren, 1970). Voluntary eye movements toward a sound source may facilitate sound localization accuracy (Platt \& Warren, 1972), whereas prevention of eye movements or movements in the opposite direction may inhibit accuracy (Jones \& Kabanoff, 1975). In dichotic listening tasks, subjects have been observed to direct their gaze in the direction of the attended channel, and they have reported that they deliberately did so in order to enhance performance (Gopher \& Kahneman, 1971; see also Gopher's study, cited in Kahneman, 1973). Moreover, selective attention in other auditory tasks appears to improve when subjects can visually fixate the sound source (Reisberg, 1978).

A particularly compelling demonstration of the phenomenon of "listening where we look" was reported recently by Reisberg, Scheiber, and Potemken (1981). In their experiment, the subjects were asked to attend to one of two word lists played simultaneously over three identical vertically arrayed speakers. The subjects showed much better target-list (correct) recall and fewer distractorlist (intrusion) errors when fixating the target speaker or a silent (control) speaker than when fixating the distractor speaker. These data were taken to suggest that eye position information is effective in directing auditory localization and attention. Reisberg et al. hypothesized that eye position information serves to help guide auditory attention by permitting the construction of a spatial

This research was performed in partial fulfillment of the requirements for an empirical honors thesis by Nigel Wolters in the Psychology Department at Oberlin College. The data were originally presented at the 1986 meetings of the Eastern Psychological Association, in Washington, DC. We wish to thank Lester Krueger, John Flowers, and Willard Larkin for their very helpful suggestions regarding the manuscript. Correspondence should be addressed to Diane J. Schiano, Psychology Department, Oberlin College, Oberlin, OH 44074. representation (or "search-image") in memory, against which the direction of auditory input can be matched.

There are two major theoretical explanations of visual facilitation effects in the auditory localization literature. These explanations differ largely in terms of the relative importance assigned to "visual" (information in the visual field) as opposed to "motor" (eye movement and position information) cues (see Mastroianni, 1982; Shelton \& Searle, 1980). Warren's (1970; see also Platt \& Warren, 1972) influential visual frame of reference theory states that a (freely viewed) textured visual field provides the information required to anchor spatial attention and hence auditory localization. Alternatively, Jones's (1975; see also Jones \& Kabanoff, 1975) spatial memory hypothesis holds that position information from the motor activity of the eyes is alone both necessary and sufficient to provide the hypothesized spatial framework in memory required for sound localization. While Reisberg et al.'s (1981) results appear to demonstrate the influence of motor cues, their methodology did not permit the direct isolation of the mechanism(s) responsible for the observed effect, since visual, motor, and even cognitive cues may have been confounded with fixation position in their study.

A close examination of the Reisberg et al. (1981) results suggests that motor position information alone clearly could not have determined the selection of auditory input in their study. For one thing, equivalent results were obtained with fixation on the target and on the silent speaker. For another, even with fixation on the distractor speaker, almost two-thirds of the responses were still from the correct (target-speaker) input. The latter finding reflects the common observation that viewing conditions may moderate, rather than determine, auditory selection. Thus Reisberg et al.'s eye position hypothesis applies only to the visual facilitation effect. Even so, it cannot easily explain why fixation on the target and on the silent speaker produced equivalent results, while fixation on the distractor speaker resulted in much poorer performance. This pattern of results required Reisberg et al. essentially to offer an explanation of visual "inhibition" rather than "facilitation," and to take the rather awkward position that look- 
ing toward an incorrect (distractor) source disrupts performance, whereas looking toward the correct (target) source may well not help at all. Since findings in the literature on the visual facilitation of auditory performance are notoriously unreliable (see Mastroianni, 1982), the robustness of results in this field must be firmly established before their real theoretical import can be assessed. In the present study, two experiments were performed in an attempt to replicate the Reisberg et al. (1981) findings.

The procedure for each experiment was identical to that reported by Reisberg et al., and their results will be considered together. Experiment 2 differed from Experiment 1 only in that it was performed in a room enclosure surrounded by echo-dampening panels. This measure was taken because it had been learned that Reisberg et al.'s original study had been conducted in a soundproof chamber in order to minimize echoes (D. Reisberg, personal communication, 1987). The use of the panels was expected to help simulate more closely the actual conditions of the previous study.

\section{METHOD}

\section{Subjects}

Seventy-two Oberlin College introductory psychology students served as voluntary participants, with 36 in each of the two experiments.

\section{Materials}

The stimuli for each experiment consisted of 6 pairs of recorded word lists. Each list consisted of $\mathbf{3 0}$ random one-syllable nouns, and the designation of each list in a pair as a relevant (target) or irrelevant (distractor) stimulus was randomly determined and counterbalanced across subjects. The lists were recorded on separate channels of a SONY reel-to-reel stereo tape recorder. All lists were read with the same female voice, at equal volume, at a rate of about 1 word per sec.

Three identical 8-in. Radio Shack speakers were placed in a vertical array directly before the subject, because sound localization in the median place, though inaccurate, is possible through either pinna (Moore, 1977) or head-movement (Mills, 1972) cues. Each speaker was precisely $123 \mathrm{~cm}$ from the subject's chinrest, with the middle speaker at about eye level, and the upper and lower speakers $61 \mathrm{~cm}$ $\left(27^{\circ}\right)$ above and below it. The designation of each speaker as a target, distractor, or silent (control) sound source was counterbalanced across trials for each subject.

\section{Procedure}

Each experiment consisted of six trials, in which the subjects were tested individually. The subjects fixated the target, the distractor, or the silent speaker, each one for two of the six trials, in random order. The subjects were told to listen to the target list for a recall test immediately following the presentation. They were told to ignore the distractor list and not to write down words from this list on the recall test, should they happen to remember them.

The trials began with a ready signal from the target speaker; then the target list began. After the first target word was spoken, the remaining 29 target words were presented with 29 distractor words concurrently, at a rate of 1 per sec. In the attempt to follow the Reisberg et al. (1981) published procedure while counterbalancing the designation of lists as target or distractor stimuli, the final word on a list was not presented when that list was used as a distractor stimulus. The subjects were allowed about $45 \mathrm{sec}$ immediately after the trial to write down as many words as they could from the target list. All the subjects easily finished their recall in the allotted time. At the start of each trial, the subjects were instructed as to where to fixate their eyes, with the order of fixation (upper, lower, middle) randomized and counterbalanced across trials. All the subjects were watched in order to ensure that they complied with the eye-position instructions and that their chins remained on the chinrest.

Experiment 1 was performed in a large, open laboratory space. Experiment 2 took place in a small room enclosure, which was surrounded on all sides by 3 -in. thick SONEX echo-proof, soundattenuating panels, in order to minimize possible echo effects.

\section{RESULTS}

Table 1 presents the results reported by Reisberg et al. (1981; see Table 1, section A), as well as the results of Experiments 1 and 2 (see Table 1, sections B and C), in terms of mean percentage correct (target) recall, intrusion (distractor) errors, and nonintrusion errors, for each fixation condition. As can clearly be seen in the table, Reisberg et al. reported high correct recall rates, and levels of intrusion lower than those of nonintrusion errors. More importantly, however, Reisberg et al. observed significant effects of eye position on recall and on intrusion errors in their experiment. Specifically, significantly higher recall and lower intrusion rates were reported with fixation on either and the target speaker or the silent speaker than were reported with fixation on the distractor speaker. However, no significant difference was found between fixation on the target speaker and fixation on the silent speaker, whether for recall or for intrusion errors.

The findings of the present studies-like those of Reisberg et al.- - show relatively high correct recall rates overall, and rates of intrusion errors somewhat lower than those of nonintrusion errors. However, the results of two overall analyses failed to demonstrate any effect of eye position on recall in these experiments.

The first analysis was performed to examine the effect of eye position on total error rates in each experiment (the same information as correct recall). No significant main

Table 1

Mean Percent Correct Recall, Intrusion Errors and Nonintrusion Errors as a Function of Eye-Fixation Position in Three Experiments

\begin{tabular}{|c|c|c|c|}
\hline & \multicolumn{3}{|c|}{ Speaker at Which Eyes Were Directed } \\
\hline & Target & Silent & Distractor \\
\hline \multicolumn{4}{|c|}{ A. Reisberg, Scheiber, and Potemken (1981) } \\
\hline Correct recall & 71.8 & 72.9 & 64.5 \\
\hline Intrusion errors & 9.3 & 10.5 & 16.1 \\
\hline Nonintrusion errors & 18.9 & 16.6 & 19.4 \\
\hline \multicolumn{4}{|c|}{ B. Experiment 1: No Echo-dampening } \\
\hline Correct recall & 60.3 & 64.6 & 65.3 \\
\hline Intrusion errors & 18.0 & 11.6 & 12.6 \\
\hline Nonintrusion errors & 21.7 & 23.8 & 22.0 \\
\hline \multicolumn{4}{|c|}{ C. Experiment 2: Echo-dampening } \\
\hline Correct recall & 64.6 & 62.1 & 61.5 \\
\hline Intrusion errors & 13.1 & 15.4 & 10.9 \\
\hline Nonintrusion errors & 22.3 & 22.5 & 27.6 \\
\hline
\end{tabular}


effect of eye position $[F(2,140)=0.65$, n.s. $]$ was found. Moreover, despite the improved echo-dampening conditions in Experiment 2, no effect of experiment $[F(1,70)=$ 0.03, n.s.] and no eye position $\times$ experiment interaction $[F(2,140)=0.63$, n.s. $]$ was demonstrated.

In the second analysis, the relative proportions of intrusion and nonintrusion errors for each eye position in each experiment were compared. A significant main effect of error type was found, reflecting the fact that nonintrusion errors rates were higher than intrusion error rates $[F(1,70)=51.03, p<.01]$ overall. No further significant effects were found. Thus, again, no main effect of eye position $[F(2,140)=2.31$, n.s. $]$ or of experiment $[F(1,70)=0.15$, n.s. $]$ was demonstrated. Moreover, no significant interactions for error type $\times$ experiment $[F(1,70)=0.21$, n.s. $]$, eye position $\times$ experiment $[F(2,140)=0.61$, n.s.], error type $\times$ eye position $[F(2,140)=1.02$, n.s. $]$, or error type $\times$ eye position $\times$ experiment $[F(2,140)=1.39$, n.s. $]$ were found.

\section{GENERAL DISCUSSION}

The pattern of results of these two experiments is clear. Despite the use of procedures highly similar to those reported by Reisberg et al. (1981), we were unable to demonstrate any effect of eye position on auditory memory. The findings of Experiments 1 and 2 did not differ, even with the echo-dampening precautions taken in Experiment 2.

Although it might be argued that a soundproof chamber would have been more effective in eliminating auditory echo location cues than the paneled room enclosure used in Experiment 2, there is little reason to expect that these cues would vary with eye position in such a way as to influence the visual facilitation (or rather, inhibition) effect that is of interest here. Moreover, it should be noted that we ran another 72 subjects in various pilot conditions that were very close (though not exact) replications of the Reisberg et al. (1981) procedure, with results similar to those of the present experiments: that is, no significant effects of eye position were found.

A number of studies (e.g., Skavenski, Haddad, \& Steinman, 1972; Stevens et al., 1976) have demonstrated that the afferent signal from the eye muscles does not in itself determine subjects' visual localization abilities (especially when it conflicts with information from other sources; most notably, with efferent signals). While the present studies are clearly not definitive on this issue, their null findings-together with the results in the visual domainsuggest that sensed eye position may well not be sufficient to produce the highly precise localization of atten- tion required for the observation of effects in a difficult auditory task of the type developed by Reisberg et al. (1981).

In an important critical review of the field, Mastroianni (1982) has observed that "the literature on intersensory facilitation is riddled with contradictory findings and with failures to replicate earlier findings." Since procedural differences can contribute to variability in results, close replications of previous procedures are needed in this field in order for the robustness of experimental findings to become firmly established. The complete soundproofing conditions of Reisberg et al.'s study were admittedly not used in the present research. Yet the clear results of these experiments suggest that the oft-cited effect of eye position on performance in this task, if replicable at all, must be very fragile indeed.

\section{REFERENCES}

Gopher, D., KahNeman, D. (1971). Individual differences in attention and the prediction of flight criteria. Perceptual \& Motor Skills, 33, 1335-1342.

JoNES, B. (1975). Visual facilitation of auditory localization in schoolchildren: A signal detection analysis. Perception \& Psychophysics, 17, 217-220.

Jones, B., \& KaBANOFF, B. (1975). Eye movements in auditory space perception. Perception \& Psychophysics, 17, 241-245.

Kahneman, D. (1973). Attention and effort. Englewood Cliffs, NJ: Prentice-Hall.

MastroianNi, G. R. (1982). The influence of eye movements and illumination on auditory localization. Perception \& Psychophysics, 31, 581-584.

MiLLS, A. (1972). Auditory localization. In J. Tobias (Ed.), Foundation of mocern auditory theory (Vol. 2, pp. 303-348). New York: Academic Press.

MoORE, B. (1977). The psychology of hearing. New York: Macmillan.

Platt, B. B., \& WarRen, D. H. (1972). Auditory localization: The importance of eye movements and a textured visual environment. Perception \& Psychophysics, 12, 245-248.

ReISBERG, D. (1978). Looking where you listen: Visual cues and auditory attention. Acta Psychologica, 42, 331-341.

Reisberg, D., Scheiber, R., \& Potemken, L. (1981). Eye position and the control of auditory attention. Journal of Experimental Psychology: Human Perception \& Performance, 7, 318-323.

Shelton, B. R., Searle, C. L. (1980). The influence of vision on the absolute identification and sound-source position. Perception \& Psychophysics, 28, 589-596.

Skavenski, A. A., Haddad, G., \& Steinman, R. M. (1972). The extraretinal signal for the visual perception of direction. Perception \& Psychophysics, 11, 287-290.

Stevens, J. K., Emerson, R. C., Gerstein, G. L., Kallos, T., NeuFeld, G. R., Nichols, C. W., Rosenquist, A. C. (1976). Paralysis of the awake human: Visual perceptions. Vision Research, 16, 93-98.

WARREN, D. H. (1970). Intermodality interactions in spatial localization. Cognitive Psychology, 1, 114-133.

(Manuscript received April 15, 1988; revision accepted for publication August 3, 1988.) 